\title{
Paper Self-organized Clustering for Improved Interference Mitigation in White Spaces
}

\author{
Julio Aráuz ${ }^{1}$ and Alberto Sánchez ${ }^{2}$ \\ ${ }^{1}$ School of Information and Telecommunication Systems, Ohio University, USA \\ 2 Colegio de Ciencias e Ingenierías, Universidad San Francisco de Quito, Quito, Ecuador
}

\begin{abstract}
In this paper a collaborative coexistence mechanism for white space base stations is proposed. We look at the case where these base stations operate in geographical areas where the density of used TV channels is such that only one channel is left for broadband access. We show how with cooperative closed loop control and a clustering strategy, it is possible to find feasible power assignments that provide a flexible and stable coverage solution. The framework under which we study our proposal is based on the IEEE 802.22 standard, which provides white space guidelines for applications in broadband access or machine-to-machine communications. We propose and evaluate a self-organized, collaborative power control and design strategy to enable effective coexistence of base stations under extreme bandwidth constraints. Finally, we also portray how proposed approach positively compares against others from different wireless access technologies.
\end{abstract}

Keywords-interference mitigation, self-coexistence, self-organized, white spaces, wireless.

\section{Introduction}

The operation of cognitive white spaces networks that employ unoccupied television bands is already allowed in the USA and the UK. Other countries are considering opening up this part of the spectrum as well. In the USA, the Federal Communications Commission (FCC) established the requirements for the use of unlicensed spectrum by television band devices (TVBDs) [1]. TVBDs are secondary users of spectrum where TV stations or wireless microphones might already be present. To cope with possible interference with incumbent users, the FCC originally mandated two main control methods one based on a geographical incumbent spectrum database and a second one, now optional, based on spectrum sensing of incumbent users.

On the standardization side, the IEEE has dealt with white spaces technology in two significantly different approaches, the 802.11af and 802.22 standards. The 802.11af standard, approved in 2014, is an Orthogonal Frequency Division Multiplexing (OFDM) based approach geared towards short links with lengths in the order of units of kilometers. A noteworthy advantage of the 802.11af standard is that it is able to take advantage of the solidly established 802.11 manufacturing and certification capabilities. This is expected to enhance its leverage over other competing standards. On the other hand, the 802.22 standard, approved in 2011, focuses on providing services over much larger areas referred to as Wireless Regional Area Networks (WRAN), which can span tens of kilometers [2]. Other industry forums have also worked on separate standardization efforts [3]. In this article, we focus our study on the capabilities provided by the IEEE 802.22 standard.

In white space networks, TVBDs are designed to dynamically search idle spectrum and provide infrastructure-based coverage using VHF or UHF bands. These bands possess very appealing propagation characteristics as, under equal conditions, signals can travel further in comparison to cellular bands that typically employ higher frequencies. In 802.22 networks, cells could be as large as or larger than those of a legacy cellular network. The usable spectrum in these bands may vary widely depending on the location of incumbent users [4]. Both TV stations and wireless microphones, which are primary incumbent users, are likely to be found near urban or densely populated suburban areas affecting the available bandwidth for TVBDs.

In this work, we are interested in looking at the case where TVBDs provide coverage to a particular area when just one channel is available. In particular, we consider USA TV channels that use $6 \mathrm{MHz}$ of bandwidth. In a previous research effort, we referred to this scenario as a dense case. In such scenario, the TVBDs are expected to operate in an area with a high density of already occupied TV channels [5]. In this article, we place emphasis on three significant aspects of white space networks. First, we study if it is possible to effectively deploy these networks under extreme bandwidth and interference constraints in a selforganized but low complexity manner. Second, we look at the overall capacity that the network offers under scarce bandwidth conditions. Finally, we discuss how this proposal compares against schemes proposed for other access technologies like Long Term Evolution (LTE).

The work we discuss next differs from our previous efforts as here we base our solution on a clustering scheme to autonomously group base stations serving a given geographical area. We found that clustering enhances system behavior and performance as it allows better reuse of spectrum and results in larger amounts of bandwidth available for the TVBDs. We also propose a new autonomous self-characterization method for interference mitigation and 
a novel mechanism to cover users in remote areas with an autonomously selected macro base station (BS). Finally, we provide an analysis that illustrates stability and convergence of our proposal. These additions to presented analysis contrast with our previous work in the area where we dynamically tuned metrics to control interference without taking into consideration any clustering, coverage of remote users or stability conditions [5], [6].

In the remainder of this article, we will first discuss the motivations behind our work and then review related research in the area. Thereafter, we will visit the generalities behind the IEEE 802.22 standard and place particular attention on its self-coexistence mechanism. We then proceed to describe proposed approach, a formal stability and convergence analysis, and an optimum solution. We close with a performance evaluation of our results and a discussion contrasting our findings with other possible alternatives.

\section{Motivation}

In the U.S. the FCC mandated the creation of a national database that can be used by TVBDs to determine bandwidth availability based on their geographical location. Since we are interested in looking at the dense case, we needed more detailed information than what is presented in previous studies [4]. Therefore, we used one of the currently approved databases for incumbent users to evaluate the bandwidth availability in the downtown areas of the biggest U.S. cities [7]. We found out that close to these areas there are regularly few or no free TV channels available. This suggests that white space networks are going to be more attractive to operators in suburban or rural areas where fewer incumbent users may be present. In analysis, we looked at the bandwidth availability in regions located $16 \mathrm{~km}$ from the downtown areas of large cities. The results are presented in Table 1.

Our findings show that it is common to have a limited number of unoccupied channels in suburban areas where millions of people reside. Moreover, these channels might in some cases become unavailable due to wireless microphone usage augmenting the problem of bandwidth scarcity. Therefore, in these critical cases, it is vital to count with effective bandwidth sharing and interference mitigation

Table 1

Number of unoccupied TV channels for the five largest cities in the U.S. at a $16 \mathrm{~km}$ (10 mile) radius from the corresponding downtown area

\begin{tabular}{|l|c|}
\hline \multicolumn{1}{|c|}{ City } & Unoccupied channels \\
\hline \hline New York & 1 \\
\hline Los Angeles & 1 \\
\hline Chicago & 1 \\
\hline Houston & 8 \\
\hline Philadelphia & 2 \\
\hline
\end{tabular}

mechanisms that enable operation under spectrum availability constraints.

\section{Related Work}

Numerous optimization techniques have been proposed to mitigate interference in infrastructure networks. Usually the objective of these optimization approaches is to guarantee coverage or increase throughput [8]. These techniques commonly employ applications of water filling algorithms to find feasible solutions. While these approaches are obviously powerful, they lack practicality for distributed solutions as base stations have limited computational resources. It is feasible to find closed solutions for managing interference among wireless stations given the spatial distribution of the transmitters in an area. For instance, using Perron's theorem, which characterizes eigenvalues for a set of matrices with non-negative entries, the transmission power for $n$ mobiles nodes can be computed to meet a signal to noise ratio (SNR) objective at the base stations. This method allows finding a feasible solution with positive transmission powers for all nodes in an area given just the path losses between them. However, this mechanism may not find a feasible solution when there is excessive noise in the system [9]. In the past we have compared the results from this approach with one that employs a self-organizing procedure where power control is used to limit coverage and thus mitigate interference [5], [6].

With the introduction of femto and small cell technology, 3GPP has also looked at interference mitigation in heterogeneous environments. Two main techniques have been considered for the standards body [10], [11]. The first one, employed for user data, is known as inter-cell interference coordination (ICIC) and makes use of the flexibility of orthogonal frequency division multiple access (OFDMA) to assign different subchannels to serve users located at edge cells employing fractional frequency reuse (FFR). The second approach, used for control signals, is known as enhanced ICIC (eICIC). This latter approach includes solutions in the power and the time domain. In the power domain, interference is mitigated with power control. In the time domain, it is mitigated by hindering nodes from transmitting at the same time.

Previous work has also shown how using artificial intelligence frameworks based on reinforcement learning theory it is possible to perform interference mitigation. For example, Q-learning is one of the approaches that can be parameterized to rely only on local information to carry out interference management without the need for network nodes to exchange information between them [12]. In such an approach per node policies can be employed to map a measured interference map to a reward with the goal of iteratively increasing the reward as interference decreases. To achieve the goal, nodes first select a subcarrier allocation and then employ a separate convex optimization method to calculate the power allocation. Although the approach has been shown to be effective, we believe that dynamically 
solving the optimization problem at a base station that typically has limited computational resources is a non-trivial challenge. Other separate efforts have also employed Qlearning based solutions as well. In these studies, the solutions rely on finding subchannel allocations for the femtocells in a way that interference is mitigated by frequency diversity. These approaches are basically formal implementations of LTE's ICIC [13], [14].

It is also possible to enhance interference mitigation in white space networks by going beyond the information available about spectrum availability in a regulator's approved databases. For instance, recent research efforts employ a white space prediction algorithm to compute the minimum distance that must exist between white space operators and incumbent users [15]. The algorithm works by iteratively reducing the transmission power of white space devices until pre-defined interference mitigation goals at the contour of the incumbent TV operator are reached. Such an approach could result in significant increases of bandwidth availability, especially in geographical zones previously excluded via regulation [15].

For the 802.22 standard, interference mitigation efforts have focused on enhancing spectrum-sensing mechanisms to allow the operation of white space devices in locations where incumbents operators are present. Sensing can be carried out by spectrum managers in either centralized or distributed approaches. The trade-off between these two being accuracy and required overhead. Centralized solutions, where a single spectrum manager carries out spectrum sensing for a region, will result in lower accuracy. On the other hand, distributed solutions, where users carry out the sensing, will require a higher management overhead. It is also feasible to construct a mixed solution where a spectrum manager cooperates with geographically distributed users to determine spectrum availability. In such a case, it is possible to simplify sensing by using location and propagation estimations to select a subset of all the users to potentially minimize the use of correlated fading data and thus reduce management overhead [16].

In regards to coexistence of multiple white space operators in a given geographical area, it is possible to employ the procedures defined in the IEEE 802.19.1 standard [17]. This standard enables a system where white space operators register with a coexistence discovery and information service (CDIS). The system automatically directs operators to employ different available TV channels whenever possible, thus avoiding throughput degradation due to co-channel interference [18].

A different approach to coexistence is to use an on-demand spectrum contention (ODSC) approach, where a white space operator transmitting on a given channel cooperates with other operators by sharing its spectrum based on bandwidth requests received from other parties. In ODSC any contention for bandwidth is resolved with a mechanism based on a random procedure, referred to as a contention priority number (CPN) which is implemented inside the bandwidth requests [19]. However, ODSC has been found to be open to misuse by operators that can unfairly assign high priority to their bandwidth requests. Changes in the CPN assignment procedure have been shown to result in increased robustness against misuse [20].

Presented proposal needs to diverge from the existing cellular network related approaches as white space networks are fundamentally different from LTE based heterogeneous networks for three main reasons. First, white space networks operate in unlicensed spectrum. The ultimate goal behind unlicensed operation is promoting high rate of adoption and device affordability through competition to cover large areas. This means that the feature set to be implemented should be fundamentally simple. Second, white space networks operate using lower frequencies than those traditionally employed in cellular networks. This enables the deployment of very large cells without precluding the use of small cells. Third, TVBDs are likely going to operate under different operators (commercial, non-profit, private, etc.) with essentially different goals, policies and management capabilities. Therefore, we consider unrealistic, at least at first, to expect all devices to have similar advanced capabilities. Even if devices have similar functionalities, management policies might easily hinder operation of elaborate interference management techniques such as the use of sectorization along with FFR and autonomous frequency planning.

Additionally, our proposal provides an approach that, in contrast to related work, exploits the self-coexistence mechanisms already present in the 802.22 standard. Previous work has focused on enhancing the standard by incorporating complex signaling procedures. While this work does include the need for backhaul signaling, as we argue later, this is not critical.

\section{Preliminaries on IEEE 802.22}

In an 802.22 network, base stations (BSs) provide wireless coverage using unoccupied TV bands with cells that have a radius up to several tens of kilometers [21]. Subscribers can access the network via customer premises equipment (CPE). CPEs are expected to have outdoor directional antennas similar to those used for legacy broadcast TV reception. However, in contrast to other popular wireless technologies, no mobility is supported.

The air interface employed is OFDMA based for both the downstream (DS) and upstream (US) directions. In the dense case for white spaces, where there is only one channel available, a feature in 802.22 that is vital to maintain operation is referred to as self-coexistence. In self-coexistence mode, base stations with overlapping coverage using the same channel share the spectrum on a per frame basis. In this mode, each base station is autonomously allocated a subset of frames from a 16 frame superframe structure as shown in Fig. 1. Then each BS and the CPEs in a WRAN cell transmit only during their active frame(s) allocated in the superframe. 


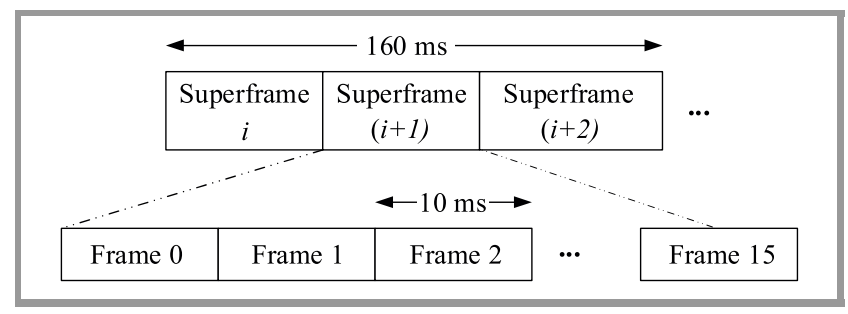

Fig. 1. Superframe structure in 802.22 .

The self-coexistence operation in 802.22 relies on channel monitoring and on the coexistence beacon protocol (CBP). CBP uses beacon packets regularly transmitted by the BS over the air or through a backhaul link to allow, among other things, the dissemination of information of frame reservation patterns and frame structure in neighboring cells. In particular, a BS goes into self-coexistence operation when it cannot acquire an empty channel after initialization. In such a situation, the BS is forced to select a channel occupied by one or more other WRAN cells. In self-coexistence mode, all base stations involved use an on-demand frame contention mechanism to share a channel with common DS/US split. This is necessary to enable possible sharing of the superframe structure.

\section{Self Organizing Strategies for Interference Mitigation}

The goal of presented solution is to mitigate interference between BSs sharing a single channel. We propose using a three-step approach. In the first step BSs autonomously group each other into clusters. This allows us to employ similar interference mitigation schemes in BSs that coexist spatially close to each other. In a second step, the BSs cooperative control their transmission power towards individual interference mitigation goals. Lastly, the system selects a macro BS to provide coverage to those CPEs that have been left out of service as a result of the interference mitigation process.

\subsection{Autonomous Clustering}

In our work we take into consideration the fact that reducing the power of BSs that are geographically closed to each other, with the goal of creating non-overlapping cells, may result in having BSs with very small footprints. This could potentially leave a considerable fraction of CPEs unserved. Therefore, we propose using a self-organizing strategy as a first step to achieve interference mitigation. In this strategy, BSs run a clustering algorithm based on their spatial distribution and self-group themselves. The goal of employing clustering is to assign similar goals to each BS in a cluster.

In our proposal, to find the clusters we employ the k-means algorithm and run it at each base station [22]. The goal of the algorithm is to minimize the objective function $J$ shown in Eq. (1). Calculating $J$ requires $C$, the number of clusters to find, and $\mathbf{x}_{\mathbf{i}}$, a vector of Cartesian coordinates of $B S_{i}$ :

$$
J=\sum_{l=1}^{C} \sum_{i=1}^{n}\left\|\mathbf{x}_{\mathbf{i}}{ }^{(l)}-\mathbf{c}_{\mathbf{l}}\right\|^{2} .
$$

The term $\mathbf{c}_{\mathbf{l}}$ represents the centroid coordinates recomputed at each iteration. In our solution, we followed the common approach of initially selecting a random assignment of centroids within the service area. However, since all BSs need to arrive to the same clustering solution, we employ the same initial random assignment at each BS. In an actual implementation, a pre-selected BS would be chosen to compute the initial centroid assignments and then distribute this information to the other BSs.

With this procedure when the number of clusters, $C$, is known each BS can independently compute the same clustering set with knowledge of the positions of all other BSs. This can be done without the need to exchange any additional information.

We compute the number of clusters by using the gap statistic approach [23]. This method is based on a variable, $W_{l}$ that quantifies the compactness of a cluster as illustrated in Eq. (2):

$$
W_{l}=\sum_{l=1}^{C} \frac{1}{2 n_{l}} D_{l},
$$

where $n_{l}$ is the number of BSs in cluster $l$ and $D_{l}$ the sum of the corresponding intra-cluster distances among the BSs in the cluster. The gap statistic defines the number of clusters as that where $\log \left(W_{l}\right)$ is a gap that is the farthest below a null reference distribution of BSs. This reference distribution is one for which there is no obvious clustering and in our case, is generated by uniformly sampling the original set of BSs.

The downside of automating cluster size selection is an increase in complexity. The gap statistic demands centralized iterative computations, as it is still necessary to generate a set of reference distributions for each possible number of clusters and then select the one that correspond to the gap as discussed above. In our case, the possible number of clusters is bounded by the number of BS in the system.

\subsection{Cooperative Control}

In a second step we employ cooperative power control to mitigate interference following a scheme we have previously proposed [6]. For clarity, we include the details of the closed loop controller here as well.

Consider a wireless network of $n$ IEEE 802.22 base stations connected to a backhaul network. Following the standard specifications, base stations are fixed and can have a maximum transmission power $W_{i}(i=1,2, \ldots, n)$. In practice, the value of $W_{i}$ is obtained from a publicly available geolocation database [7]. Similarly, CPEs are fixed as specified in the standard. We only consider the "dense case" where one $6 \mathrm{MHz}$ channel is available in the study area. This bandwidth corresponds to that assigned to broadcast TV stations in the US. 
In this paper we look at the downlink case. We consider that each $B S_{i}$ has a transmission power denoted by $P_{i}(i=1,2, \ldots, n)$ which is dynamically computed using the controller illustrated in Fig. 2. In each controller, the coordination variable is $\gamma_{i}(m)$ and is defined as the average of the SNIR values reported by a subset of the CPEs to $B S_{i}$ in iteration $m(m=0,1,2, \ldots)$. Thus for a $B S_{i}$ receiving $R_{j}(j=1 \ldots s)$ SNIR reports from a subset of CPEs this average can be expressed as:

$$
\gamma_{i}(m)=\frac{1}{s} \sum_{j=1}^{j=s} R_{j} .
$$

The subset of $s$ CPEs considered at $B S_{i}$ is formed by those CPEs that initially reported that at their location $B S_{i}$ had the highest SNIR among all the BSs they detected. As discussed in the stability analysis section once this subset is assigned we do not change it thereafter.

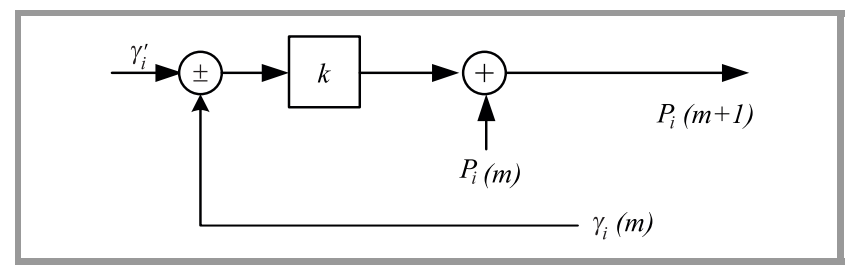

Fig. 2. Controller structure at each TVBD base station $i$.

The controller we propose has a structure similar to that of a proportional integrative (PI) one (Fig. 2). It controls the output power of $B S_{i}$ at time $t=m+1$ using following the control law:

$$
P_{i}(m+1)=P_{i}(m)-k \times\left(\gamma_{i}(m)-\gamma_{i}^{\prime}\right),
$$

where $P_{i}(m)$ and $P_{i}(m+1)$ are the transmission powers of $B S_{i}$ at iteration $m$ and $m+1$ respectively, and $k$ is the controller gain. The goal is to make $\left(\gamma_{i}(m) \rightarrow \gamma_{i}^{\prime}\right)$ where $\gamma_{i}^{\prime}$ is set following the procedure described in the next section. The goal is achieved by iteratively adjusting power at the BSs following the control law. We assume all BSs cooperate synchronously and that through regular channel quality reports have access to the corresponding CPEs SNIR. A goal is considered achieved once $\left|\gamma_{i}(m)-\gamma_{i}^{\prime}\right| \leq \varepsilon$, where $(\varepsilon \rightarrow 0)$.

\subsection{Controller Self-characterization}

After the clusters have been created the value of $\gamma_{i}^{\prime}$, used in the goals of each base station, needs to be determined. In this work, we study and compare, two heuristic based approaches that assign a value to this variable. We refer to these approaches as cluster size based and cluster and centroid based.

\subsubsection{Cluster Size Based Approach}

In this first case we mainly base the value of $\gamma_{i}^{\prime}$ on the size the cluster $B S_{i}$ is in. We employ a linear relationship of the following form.

$$
\gamma_{i}^{\prime}=\sigma r_{i}+\phi_{1}
$$

where $\phi_{1}$ represents the minimum SNIR required by a CPE to detect a signal. Notice that the selected function for $\gamma_{i}^{\prime}$ ensures that it never falls below $\phi_{1}$.

For the slope, $\sigma$, of the linear relationship we employ the following expression:

$$
\sigma=\frac{\phi_{2}-\phi_{1}}{C_{\max }-C_{\min }}
$$

where $\phi_{2}$ which is the minimum SNIR a CPE requires to operate using $16 \mathrm{QAM} 1 / 2$ as the modulation and coding scheme (MCS). This is a MCS a CPE implements as a mid-tier capability and thus we selected it as a typically desired operating region. $C_{\max }$ and $C_{\min }$ are the maximum and minimum cluster sizes in the system respectively. This information is already available to all BSs after running the $k$-means algorithm.

In sparse spatial distributions of BSs the cluster creation process may result in having a number of clusters equal to the number of BSs and thus $C_{\max }=C_{\min }$. If that is the case we set $\sigma=\phi_{2}-\phi_{1}$. However, these cases where there is no clustering are of limited interested in proposed approach. Finally, we set $r_{i}$ as follows:

$$
r_{i}=Z_{i}
$$

where $Z_{i}$ the cluster size $B S_{i}$ is in. Since the slope, $\sigma$, of the linear function defining $\gamma_{i}^{\prime}$ is always greater than zero, $\mathrm{BSs}$ in larger clusters are given higher goals. This in turn mitigates the coverage problem that results from excessive power reductions in geographically close BSs present in previous studies [5].

\subsubsection{Cluster and Centroid Based Approach}

In this second approach, we compute $\gamma_{i}^{\prime}$ with the same expression given in Eq. (5) but set the value of $\sigma$ as:

$$
\sigma=\frac{\phi_{2}-\phi_{1}}{C_{\max }-\left(C_{\min } \times\left\langle d_{\min }\right\rangle\right)},
$$

where $d_{\min }$ is the minimum of all the distances from a BS to the centroid of the system. The notation $\langle d\rangle$ represents the normalizing operation of a distance $d$ from a BS to the centroid of the system to the maximum of all these distances.

In this second approach we set $r_{i}$ as:

$$
r_{i}=Z_{i} \times\left\langle d_{i}\right\rangle,
$$

where $d_{i}$ is the distance of $B S_{i}$ to the centroid of the system of BSs. With this approach, we achieve giving BSs in larger clusters and further away from the centroid higher goals. The reason being that BSs located further away from the centroid are less likely to cause interference when assigned higher goals. 


\subsection{Autonomous Selection of a Macro BS}

As the BSs cooperate to adjust their transmission power towards achieving the goal they may eventually leave some CPEs in border areas without coverage. At that point, rather than just stopping the control loops we execute a third step guaranteeing coverage at border areas [5]. For this additional step, we take into consideration that any BS possesses the location information of all the BSs in the system making it possible to distributively compute the centroid of the system and thus identify the BS closest to the centroid. Thereafter the system can autonomously select the BS closest to the centroid as a macro BS allowing it to provide coverage to CPEs located far away from BS clusters by transmitting at a higher or maximum power. In the work presented here, we always make the decision to activate one BS as a macro coverage one.

In our particular study, we always let all BSs go through the power control loop and achieve their goals as then we can quantify what fraction of CPEs will lie in an uncovered area. In any case our approach improves coverage at the expense of having all non-macro BS in the area having to share superframe bandwidth with the macro BS. While the percentage of frames from the superframe assigned to the macro BS could be based on traffic demands, without loss of generality, in this paper we employ a static approach when assigning superframe capacity to the macro BS.

\section{System Analysis}

\subsection{Framework}

We analyze our autonomous approaches via simulation using two BS placement configurations sets for providing coverage to a given geographical area. In configuration "a" we randomly place BSs at a distance where the path loss between them is $100 \mathrm{~dB}$. In configuration "b" we place them in locations where the average path loss is $88 \mathrm{~dB}$.

The heuristically chosen path loss values allow us to study the effects of clustering on interference mitigation. In configuration "a", BSs are placed in locations where they operate $10 \mathrm{~dB}$ away from a typical value of a commercial receiver sensitivity $(-110 \mathrm{dBm})$ but can still detect each other. In configuration "b", all BSs operate well within the detection range of all other BSs.

For propagation losses, we use the Egli model. This legacy model was designed from experimental measurements in the UHF and VHF bands taken in the East Coast of the USA [24]. The model only takes into account experimental terrain irregularities. We selected this model as it enables, without loss of generality, the simple closed solution stability analysis presented in the next section. Using the Egli model we can express, $P_{i j}$ which is the median received power in watts at $\mathrm{CPE} j$ from $B S_{i}$ as:

$$
P_{i j}=G_{t x} G_{r x} \times\left(\frac{h_{t x} h_{r x}}{d_{i j}^{2}}\right)^{2} \times\left(\frac{40}{f}\right)^{2} \times P_{i},
$$

where $G_{t x}$ and $G_{r x}$ are the antenna gains of the transmitter (BS) and receiver (CPE) respectively, $h_{t x}$ and $h_{r x}$ are their corresponding antenna heights in meters, $d_{i j}$ is the distance between $B S_{i}$ and CPE $j, f$ represents the carrier frequency expressed in $\mathrm{MHz}$, and $P_{i}$ is the transmission power of $B S_{i}$ expressed in watts. The numerical value 40, which has as units the reciprocal of those of $f$ is employed when median received powers are desired as output. The experimental curves developed by Egli also allow computing path losses other than those corresponding to the median one; this is done by adjusting the result with a frequency dependent variable [25]. In Table 2 we expand the corresponding model parameters and levels we employ in this analysis.

Table 2

Simulation study parameters

\begin{tabular}{|l|c|}
\hline \multicolumn{1}{|c|}{ Parameter } & Level \\
\hline \hline Study area & $10 \times 10 \mathrm{~km}$ \\
\hline Max BS transmission power & $30 \mathrm{dBm}$ \\
\hline TV channel bandwidth & $6 \mathrm{MHz}$ \\
\hline BS antenna height $\left(h_{t x}\right)$, gain & $15 \mathrm{~m}, 12 \mathrm{dBi}$ \\
\hline CPE antenna height $\left(h_{r x}\right)$, gain & $-110 \mathrm{dBm}$ \\
\hline CPE and BS sensitivity & $5 \mathrm{~dB}$ \\
\hline Noise figure & 20 \\
\hline Number of runs per experiment & 0.001 \\
\hline$\varepsilon$ & $\{5\}$ \\
\hline Number of BSs $(n)$ & $\{20,40,60, \ldots, 120\}$ \\
\hline Number of CPEs $(q)$ & $6 \mathrm{~dB}, 10 \mathrm{~dB}$ \\
\hline$\phi_{1}, \phi_{2}$ & $10 \%, 20 \%, \ldots, 90 \%$ \\
\hline $\begin{array}{l}\text { CPE density around } 1.6 \mathrm{~km} \\
\text { of the BS }\end{array}$ & $10 \%$ \\
\hline
\end{tabular}

\subsection{Stability Analysis and Convergence}

For the case discussed in this paper and taking into account the control law from Subsection 5.2, the system can be represented as an instantaneous gain given by the power propagation model from Eq. (10), in cascade with the SNIR

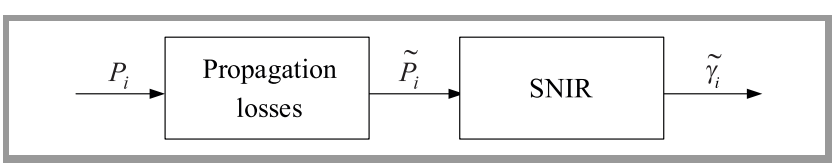

Fig. 3. Open loop system.

calculation. The diagram is presented in Fig. 3 and the resulting transfer function as:

$$
\tilde{\gamma}_{i}=\frac{G_{t x} G_{r x}}{P_{\text {noise }}+\sum_{i \neq j} P_{i j}} \times\left(\frac{h_{t x} h_{r x}}{d_{i j}{ }^{2}}\right)^{2} \times\left(\frac{40}{f}\right)^{2} \times P_{i} .
$$




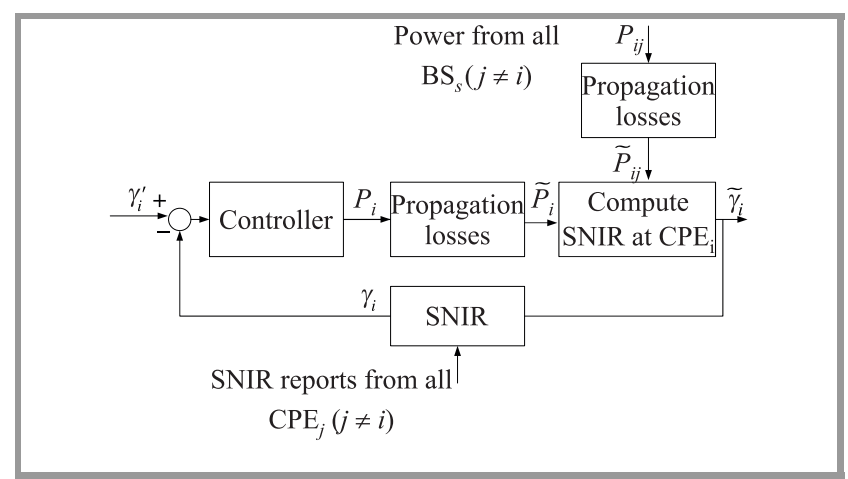

Fig. 4. Closed-loop self-organized interference mitigation system.

For simplicity, we collect all of the contributions from CPEs associated to a BS which have the form from Eq. (11) in a single gain $M_{i}(z)$ defined as:

$$
M_{i}(z)=\frac{G_{t x} G_{r x}}{P_{\text {noise }}+\sum_{i \neq j} P_{i j}} \times\left(\frac{h_{t x} h_{r x}}{d_{i j}{ }^{2}}\right)^{2} \times\left(\frac{40}{f}\right)^{2} .
$$

It is important to notice that $M_{i}(z)$ will change its value at each iteration given that the power terms $P_{i j}$ will be updated simultaneously. In the system, CPEs that report their SNIR to a BS are selected at initialization and not reassigned thereafter. Under these assumptions, and considering that due to clustering each CPE reports SNIR values similar to each other, the control loop can be modeled as shown in Eq. (13). A schematic diagram of the complete system is shown in Fig. 4.

$$
\gamma_{i}=k \times \frac{M_{i}(z)}{\left(1-z^{-1}\right)+k M_{i}(z)} \times \gamma_{i}^{\prime}
$$

Therefore, the control loop will be asymptotically stable as long as the closed-loop poles of the system lie within the unit circle. An important condition, for further analysis, which is not examined here, is how the computation of $\gamma_{i}$ can affect the stability of the loop. The use of the average SNIR, $\gamma_{i}$, as the feedback signal could lead to erratic behavior and even instability, especially if the variance in the measurements is very high. To avoid this condition, we bound the maximum and minimum transmission powers and once these are reached at a BS we stop the corresponding power control loop. The assumption of considering that the reported SNIR's from the nearby CPEs are similar to each other is important since if this is not met the modeled system would not be linear. Given the conditions in signal size discussed (small signal analysis), there is only local stability.

\subsection{Signaling Impact}

Regarding signaling overhead presented approach requires each BS at initialization to exchange location messages with all other BSs. This operation in a mesh connected topology will require up to $n / 2 \times(n-1)$ messages over the air or the backhaul. As the number of BSs to cover a wide area region is expected to be low due to the propagation characteristics, we do not foresee this as a limitation.

The overhead required during the self-organized power control execution is part of channel quality signaling, information that is already regularly available over the air interface. However, how fast an autonomous solution is found depends on the rate at which this signaling is exchanged. In previous related work, it was discussed how the number of iterations required for a typical loop to find a solution was on average 32 [5]. In each iteration, all CPEs associated to a BS need to send feedback information. However, as the standard does not support mobility once a solution is found there is no need to constantly reevaluate the conditions unless changes occur in the number of devices in the network.

\subsection{Optimal Solution}

To compare presented solution to an optimal baseline we developed a mixed integer linear program. This is necessary as the cooperative control strategy finds a power allocation that is non-optimal in terms of coverage. The goal of the program is to minimize the number of BSs that a CPE potentially receives service from and thus minimizes the number of overlapping coverage areas. For clarity, we also include here our previously proposed linear program for the optimal solution [5].

For a system with $n$ BSs and $q$ CPEs, consider the variable $y_{i j}$ as:

$$
y_{i j}= \begin{cases}1, & \text { if } B S_{i} \text { covers } C P E_{j} \\ 0, & \text { otherwise }\end{cases}
$$

Minimize

$$
\sum_{i=1}^{n} \sum_{j=1}^{q} y_{i j}
$$

Subject to:

$$
\begin{gathered}
\alpha_{i j} \times P_{i} \geq \delta y_{i j}, \text { for } i=1,2, \ldots, n \text { and } j=1,2, \ldots, q, \\
\sum_{i=1}^{n} y_{i j} \geq 1, \quad \text { for } j=1,2, \ldots, q, \\
P_{i} \leq W_{i}, \quad \text { for } i=1,2, \ldots, n .
\end{gathered}
$$

The objective function minimizes the number of CPEs covered by multiple BSs. Inequality (15) ensures that any $C P E_{j}$ that is covered by $B S_{i}$ has a received power from $B S_{i}$ greater than or equal to its sensitivity $\delta$. The channel loss between $C P E_{j}$ and $B S_{i}$ is represented by $\alpha_{i j} . P_{i}$ is the transmission power of $B S_{i}$. Inequality (16) ensures that any $C P E_{j}$ must be covered by at least one BS. The constraint (17) limits the maximum power any BS can select.

As the proposed linear program is a variation of the setcovering problem it is not scalable with the number of CPEs 
and BSs [26]. Nevertheless, as illustrated in the results, it provides a valuable comparison basis for configurations with a small number of CPEs.

\subsection{Results}

We study the performance of the system by looking at the mean fraction of a superframe available to any BS. Higher fractions represent better bandwidth availability on a per BS basis. We analyze these fractions as function of how close the CPEs are around a BS. We first present the results as a function of the concentration of total CPEs located $1.6 \mathrm{~km}$ (1 mile) from any BS. Lower concentration of users represents less populated areas.

Figure 5 portrays the average fraction of a superframe that is available to any BS, averaged over all total number of CPEs from Table 2. The minimum power curve represents a non-desirable solution where all BS have lowered their power so they don't interfere with each other. This line represents a condition where there is very poor coverage. The curve labeled self-coex. 802.22 indicates the performance when the process detailed in the IEEE 802.22 standard is followed to assign every BS the same number of frames in a superframe. This basically avoids interference without controlling power but by controlling access to the channel over time. The line labeled MILP represents the optimal solutions found for the mixed integer linear program; notice that due to the nature of the problem only configurations tested with up to $25 \mathrm{CPEs}$ had feasible solutions found after solving the mixed integer linear program with common algorithms.

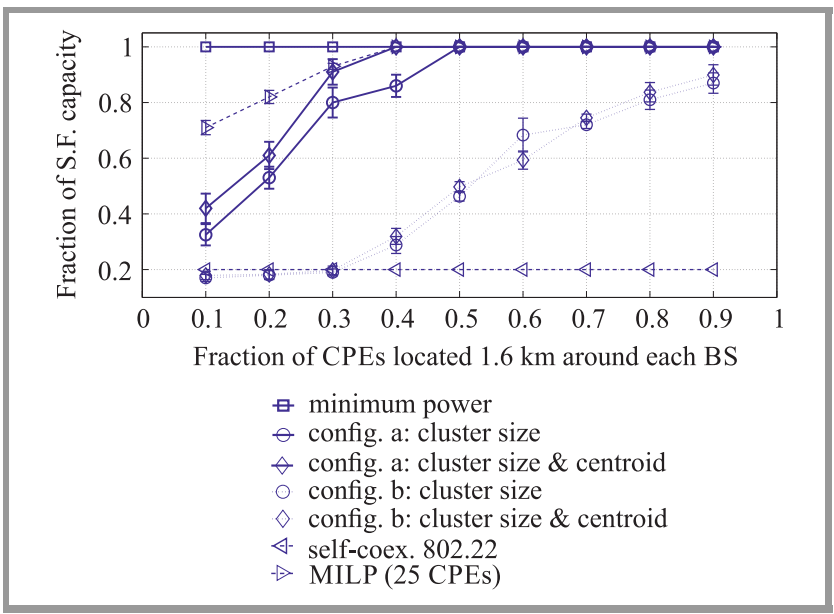

Fig. 5. Mean fraction of superframe (SF) capacity available to a BS vs. different concentration of CPEs close to the BS (averaged over all values of $q, 95 \%$ confidence intervals).

The results from our approaches in Fig. 5 are presented for the two configurations ("a" and "b") detailed in Subsection 6.1. In Fig. 5, no BS has been selected as a macro BS yet. To generate these curves after the cooperative control solution is found we compute the average number of frames in a superframe a BS has access to. A BS that does not interfere with any other BS can be assigned a whole superframe. BS interfering each other are assigned equal capacity in a superframe. Notice that for all user concentrations around the BS the average fraction of a superframe that is available has a minimum value of 0.32 compared to 0.2 of the 802.22 solution. As the user concentration grows the control strategy approaches the optimum and upper limit where no BSs interfere with each other. Equally important, the effect of considering the distance of a BS to the centroid provides an average improvement of $19 \%$ in configuration "a" where the BS are farther apart from each other. A similar general trend is observed in the results for configuration " $b$ " where the BSs are closer to each other. Naturally this makes controlling the interference more challenging and this is reflected in the fact that the curves for this configuration are always below those for the first. Nevertheless, as the user concentration increases the scheme gets closer to the optimum case of no interference. Additionally, we found that an average of only 28 iterations was needed to find a solution, similar to what we have observed in the past [5].

When CPEs are left unserved after the cooperative strategy, the BS closest to centroid can be designated as a macro BS. The effects of this condition are illustrated in Fig. 6. For this study, we assigned half of the superframe to the

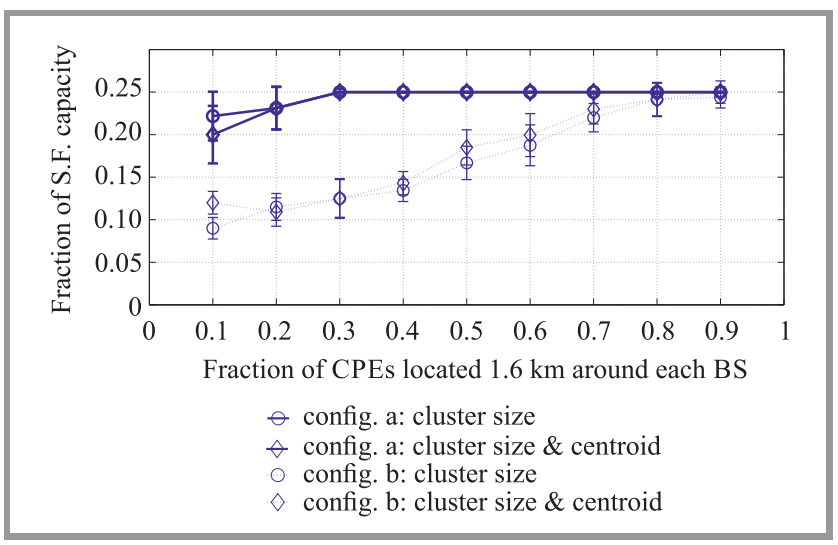

Fig. 6. Mean fraction of superframe capacity available to a BS when BS closest to centroid is assigned as a macro BS vs. different concentration of CPEs close to the BS (averaged over all values of $q, 95 \%$ confidence intervals).

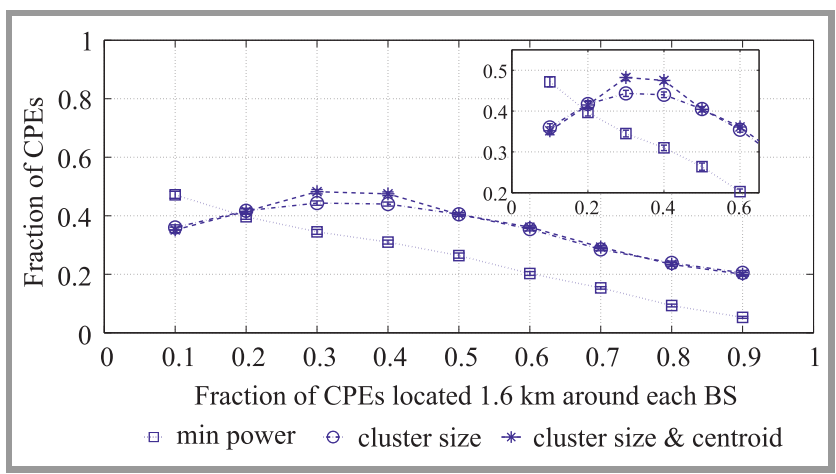

Fig. 7. Mean fraction of CPEs needing service from a macro BS for different concentration of CPEs close to the BS (averaged over all configurations and values of $q, 95 \%$ confidence intervals). 

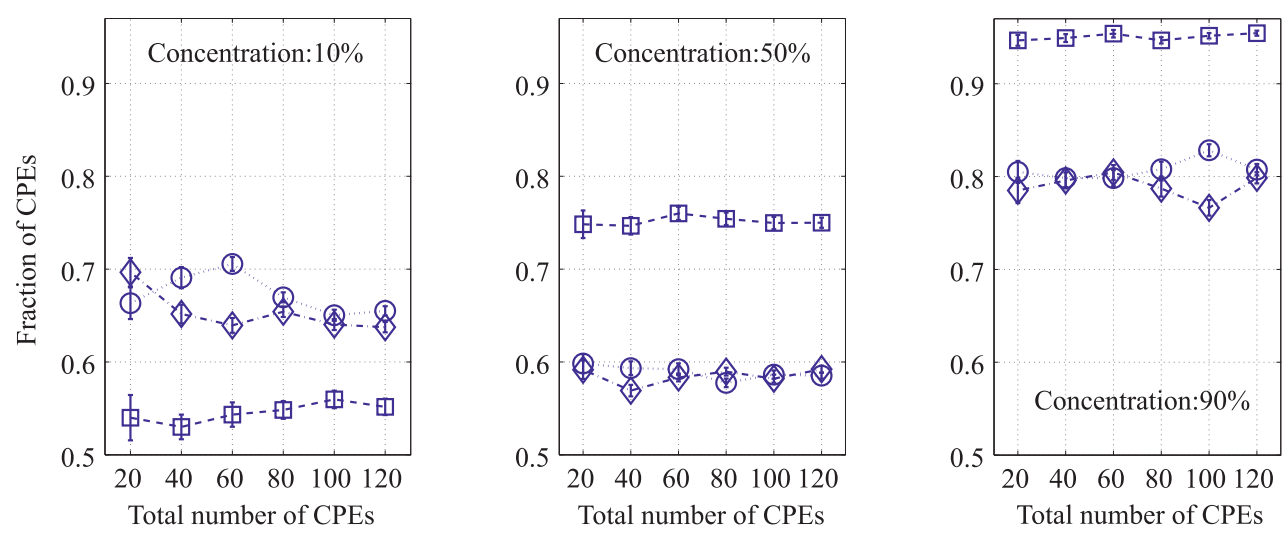

$-\square-\min$ power

cluster size $\quad \wedge$ cluster size \& centroid

Fig. 8. Mean fraction of CPEs served using different strategies for a varying number of total CPEs. Subfigures show different concentrations, 10, 50 and 90\%, of CPEs within $1.6 \mathrm{~km}$ of the BS; no selection of macro BS present (averaged over all configurations, $95 \%$ confidence intervals).

newly designated macro BS and distributed the rest of the capacity equally among the other BSs. Notice that this effectively results in solving the same problem with one less base station. In this case, the actual magnitude of the benefits depends on the fraction of bandwidth assigned to the macro BS, a decision that falls outside the scope of this paper. Designating a BS as a macro BS and giving it access to some fraction of the superframe also provides the same flexibility as ICIC in LTE, where some fraction of a frame is assigned to serve edge users. However, in this case we do not require modifications to frequency planning or cell sectorization.

To understand what the impact of selecting a BS and transforming into a macro BS is, we studied the average fraction of CPEs that would need to be served by a macro BS when the system has not yet assigned a macro BS. We show this average fraction by considering the results of averaging all experiments with a given number $q$ of CPEs in the system detailed in Table $2(q=20,40, \ldots, 120)$. As shown in Fig. 7 for low concentration of CPEs around the BSs the fraction gradually grows from 37 to $41 \%$ before reaching an inflection point where it declines. This behavior occurs because with sparse placement of CPEs (only $10 \%$ or $20 \%$ close to the BSs) after the goals are reached the BSs will tend to employ higher transmission powers to cover as many CPEs as possible. Eventually when the subset of CPEs considered for the power adjustment at each base station reaches $30 \%$ most CPEs can start to be covered with lower transmission powers and thus the fraction of them requiring service from a macro BS continuously decreases. This is quite important as after this inflection point, the load the macro BS needs to handle can be significantly reduced making more bandwidth available to BSs closer to CPEs throughout the area, increasing overall throughput.

We also look at how each strategy performs when varying the total number of CPEs $(q)$ in the coverage area. Figure 8 shows how each of the strategies behave under three dif- ferent concentration of respective users in an area located $1.6 \mathrm{~km}$ around a BS. As the concentration increases from 10 to $90 \%$ the average fraction of CPEs served by all BSs increases as more users get placed progressively closer to the transmitters. For each of the strategies the fraction of CPEs served stays approximately constant indicating that the response is in general weakly dependent on the number of CPEs in the system as the control loop does not take into account the value of $q$.

\section{Closing Comments}

Presented proposal weighs in favorably in relation to those employed in traditional cellular networks like LTE. If there is a need to assign more resources to users on cell edges this can be done just by increasing the share of frames in the supeframe the macro BS has access to. In LTE related solutions, where FFR is typically used, increases in the number of users at cell edges would require reconfiguration of the number of resources assigned to serve the edge and possibly power boosting adjustments to grow the cell, tentatively impacting frequency planning.

With proposed approach, there is no need to go through additional frequency planning; the 802.22 signaling requires no changes and dynamically adapts to our requirements. Our approach also maintains simplicity and does not place excessive overhead on the backhaul as after initialization each BS operates mainly independently.

\section{Results in Perspective and Future Work}

We looked at a simple, yet robust, strategy to find downlink power allocations for base stations in 802.22 networks. We found that our proposal yields significant benefits starting at a low concentration of users around BSs, a fea- 
ture that should be attractive to new operators. In addition, we showed the stability conditions of the system by computing its transfer function and establishing the conditions which should be met.

The structure of the control systems allows for other factors to be taken into consideration. For instance, it is possible to consider the traffic demands from CPEs and find feasible power allocations that may lead not only to improve coverage but better overall throughput while maintaining a cooperative scheme with low system overhead impact.

\section{References}

[1] FCC, "Federal Communications Commission, Part 15 TV Bands Devices, Second Report and Order and Memorandum Opinion and Order", FCC 08-260, ET Docket No. 04-186, 2008.

[2] C. Stevenson, G. Chouinard, Z. Lei, W. Hu, S. Shellhammer, and W. Caldwell, "IEEE 802.22: The first cognitive radio wireless regional area network standard", IEEE Commun. Mag., vol. 47, no. 1, pp. 130-138, 2009.

[3] Weightless Special Interest Working Group, "Weightless System Specification", 2012 [Online]. Available: http://weightless.org

[4] K. Harrison, S. Mishra, and A. Sahai, "How much white-space capacity is there?", in Proc. IEEE Int. Symp. on New Front. in Dynam. Spectrum Access Netw. IEEE DySPAN 2010, Singapore, Singapore, 2010 (doi: 10.1109/DYSPAN.2010.5457914).

[5] J. Arauz and Z. Miller, "Self-coexistence in the dense case for white spaces", in Proc. of IFIP Wireless Days (WD 2012, Dublin, Ireland, 2012 (doi: 10.1109/WD.2012.6402844).

[6] Z. Miller and J. Arauz, "Self-coexistence with autonomous target variable selection for white space devices", in Proc. of Inform. and Telecommun. Education and Res. Assoc. Conf. ITERA 2013, Cincinnati, OH, USA, 2010.

[7] White Space database [Online]. Available: http://whitespaces.spectrumbridge.com/whitespaces/home.aspx (accessed on March 10, 2017).

[8] R. Madan, S. Boyd, and S. Lall, "Fast algorithms for resource allocation in wireless cellular networks", IEEE/ACM Trans. on Netw., vol. 18, no. 3, pp. 973-984, 2010.

[9] S. Pillai, T. Suel, and S. Cha, "The Perron-Frobenius theorem: some of its applications", IEEE Sig. Proces. Mag., vol. 22, no. 2, pp. 62-75, 2005.

[10] 3GPP, "Considerations on interference coordination in heterogeneous networks", LG Electronics, TSG RAN WG1 R1-101369, 2010.

[11] 3GPP, "Summary of the description of candidate eICIC solutions", CMCC, TSG-WG1 R1-104968, 2010.

[12] M. Bennis and D. Niyato, "A Q-learning based approach to interference avoidance in self-organized femtocell networks", in IEEE GLOBECOM Workshops (GC Wkshps), Miami, FL, USA, 2010, pp. 706-710 (doi: 10.1109/GLOCOMW.2010.5700414).

[13] A. Galindo-Serrano and L. Giupponi, "Distributed Q-learning for interference control in OFDMA-based femtocell networks", in Proc. of 71st. IEEE Veh. Technol. Conf. VTC201-Spring, Taipei, Taiwan, 2010 (doi: 10.1109/VETECS.2010.5493950).

[14] A. Serrano, L. Giupponi, and M. Dohler, "BeFEMTO's selforganized and docitive femtocells", in Proc. Future Network and MobileSummit 2010 Conf., Florence, Italy, 2010, pp. 1-8, 2010.

[15] G. P. Villardi, H. Harada, F. Kojima, and H. Yano, "Multilevel protection to broadcaster contour and its impact on TV white space availability", IEEE Trans. on Veh. Technol., vol. 66, no. 2, pp. 1393-1407, 2017 (doi: 10.1109/TVT.2016.2566675).

[16] S. Biswas, S. Biswas, A. Mukherjee, and M. K. Naskar, "Cooperative sensing and allocation scheme using IEEE 802.22-Standard", in Proc. of IEEE Int. Conf. on Adv. Networks and Telecommun. Syst. ANTS 2014, New Delhi, India, 2014 (doi: 10.1109/ANTS.2014.7057246).
[17] IEEE, "IEEE Standard for Information technology - Telecommunications and information exchange between systems, Local and metropolitan area networks, Specific requirements, Part 19: TV White Space Coexistence Methods", IEEE Std 802.19.1-2014, 2014.

[18] S. Filin, K. Ishizu, F. Kojima, and H. Harada, "Implementation of TV white space coexistence system based on IEEE 802.19.1 Standard", in Proc. IEEE Conf. on Stand. for Commun. and Netw. CSCN 2015, Tokyo, Japan, 2015, pp. 206-211 (doi: 10.1109/CSCN.2015.7390445).

[19] W. Hu, M. Gerla, G. A. Vlantis, and G. J. Pottie, "Efficient, flexible, and scalable inter-network spectrum sharing and communications in cognitive IEEE 802.22 networks", in Proc. 1st Int. Worksh. on Cognit. Radio and Adv. Spectrum Manag., Aalborg, Denmark, 2008 (doi: 10.1109/COGART.2008.4509981).

[20] K. Ezirim, L. Liu, P. Ji, and S. Sengupta, "Distributed and cheatproof spectrum contention scheme for IEEE 802.22 WRAN networks", in Proc. IEEE Wirel. Commun. and Netw. Conf. WCNC 2015, New Orleans, LA, USA, 2015, pp. 1095-1100 (doi: 10.1109/WCNC.2015.7127622).

[21] IEEE, "IEEE Standard for Information Technology - CognitiveWireless RAN Medium Access Control (MAC) and Physical Layer (PHY) Specifications: Policies and Procedures for Operation in the TV Bands", IEEE Std 802.22-2011, pp. 1-680, 2011.

[22] J. MacQueen, "Some methods for classification and analysis of multivariate observations", in Proc. 5th Berkeley Symp. on Math. Statistics and Probability, Berkeley, CA, USA, vol. 1, pp. 281-290, 1967.

[23] R. Tibshirani, G. Walther, and T. Hastie, "Estimating the number of clusters in a dataset via the Gap statistic", J. of the Royal Statis. Soc., vol. 63, no. 2, pp. 411-423, 2001.

[24] J. Egli, "Radio propagation above $40 \mathrm{MC}$ over irregular terrain", Proceedings of the IRE, vol. 45, no. 10, pp. 1383-1391, 1957.

[25] J. Seybold, Introduction to RF Propagation. Hoboken, New Jersey: Wiley, 2005, pp. 141-143.

[26] C. Lee and H. Kang, "Cell planning with capacity expansion in mobile communications: a tabu search approach", IEEE Trans. on Veh. Technol., vol. 49, no. 5, pp. 1678-1691, 2000.

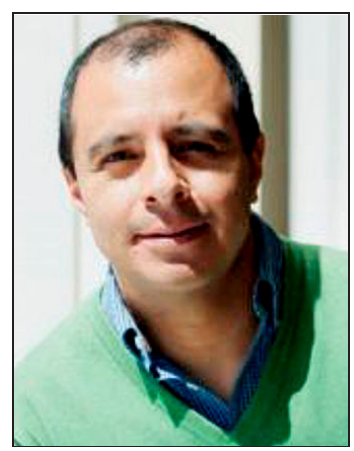

Julio Aráuz is an Associate Professor at the J. Warren McClure School of Information and Telecommunications Systems at Ohio University. He obtained both a M.Sc. in Telecommunications and a Ph.D. degree in Information Sciences from the University of Pittsburgh, USA. Before joining Ohio University he was a Research Scientist at NEC Network Laboratories in Heidelberg, Germany working for the Mobile Wireless Network Division. His research interests include wireless communications, cyberphysical and self-organizing complex systems, simulation and testbed experimentation.

E-mail: arauz@ohio.edu

J. Warren McClure School of Information and Telecommunication Systems

Ohio University

20 E. Union St.

1 Ohio University

Athens, OH 45701, USA 


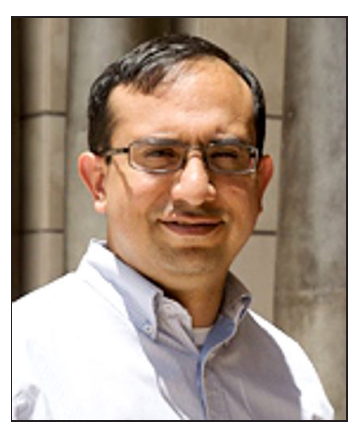

Alberto Sánchez received his B.Sc. in Electrical and Electronic Engineering from Escuela Politécnica Nacional, Ecuador; a M.Sc. from Bradford University, England, and a Ph.D. from the University of Strathclyde, Scotland. In 2011 he joined the Colegio de Ciencías e Ingenierías at
Universidad San Francisco in Quito. His research interest include reconfigurable protection systems, process monitoring, intelligent energy saving devices, collaborative systems.

E-mail: asanchez@usfq.edu.ec

Colegio de Ciencias e Ingenierías

Universidad San Francisco de Quito

Campus Cumbayá

PO-Box 17-1200-841

Quito, Ecuador 